\title{
Kluftmessungen im Gelände und ihre Bedeutung für die Bestimmung des tektonischen Spannungsfeldes in der Schweiz
}

\section{Field measurements of joints and their signific- ance for the determination of rock stresses in Switzerland}

by A. E. Scheidegger

\begin{abstract}
An intensive program of measurements of the orientation of joints in the field in Switzerland was undertaken in the Cantons of Graubünden, St. Gallen, Schwyz, Uri, Zürich, Zug, Aargau and Berne. For every «region», comprising 2-8 outcrops, the average orientation of the joint-parallelepiped was calculated by means of an electronic computer based on a function-minimization program. If it is assumed that the steeply-dipping joint surfaces have been produced by the present-day triaxial stress field according to the Mohr-Anderson theory of faulting, the orientation of the latter can be calculated. Thus, the net of present-day stress trajectories for Switzerland can be drawn. It turns out that the average orientation of the greatest compression in the region investigated is $\mathrm{N} 60^{\circ} \mathrm{W}$. This agrees well with in-situ stress determinations by Gysel in the Sonnenbergtunnel near Lucerne $\left(\mathrm{N} 76.5^{\circ} \mathrm{W}\right)$ and by Greiner in southern Germany ( $\left.28^{\circ}-50^{\circ} \mathrm{W}\right)$. Conversely, the hypothesis that most joints visible on fresh outcrops are due to the present-day tectonic stresses and that the latter is homogeneous over extended areas, is thereby confirmed.
\end{abstract}

\section{Einleitung}

Im Verfolg seiner Studien über Kluftorientierungen hatte der Schreibende Gelegenheit, während eines Aufenthaltes in der Schweiz an verschiedenen Stellen Kluftmessungen durchzuführen. Diese Kluftmessungen wurden dann nach einer von KOHLBECK und SCHEIDEGGER (1977) entwickelten Methode numerisch ausgewertet und ergaben recht interessante Resultate. Die Auswertungsmethode besteht in der Bestimmung von Dichte-Maxima von Dimroth-Watson-Verteilungen, die auf die Messwerte durch eine Maximum-Likelihood-Methode verpasst werden. Die $90 \%$ Vertrauensgrenzen werden als Fehlergrenzen angenommen.

In einem Gebiet, das tektonisch so kompliziert ist wie die Schweiz, kann die Frage nach dem Alter der gemessenen Klüfte auftauchen. Es scheint jedoch, dass Klüfte in rezenten Aufschlüssen (vor allem durch Glazialwirkung oder gar Strassenbauten freigelegte Felsböschungen) von der Schichtung oder der Genese der Gesteine in der Hauptsache unabhängig sind, sondern durch das rezente tektonische Geschehen bedingt werden. Selbstverständlich können dabei frühere Spalten und Klüfte reaktiviert werden, wenn diese zufällig nahe den durch rezentes Geschehen bedingten Richtungen liegen. In den meisten Fällen aber erkennt man, dass rezente Klüfte die älteren einfach durchschneiden.

Es wird also angenommen, dass jede Kluftschar an einem Aufschluss einer Dimroth-Watson-Verteilung folge. Der fundamentale Klüftkörper ist durch drei Kluftscharen bestimmt; man wird daher die Überlagerung von drei Dimroth-Watson-Verteilungen in den Messwerten eines Aufschlusses oder einer Gruppe von Aufschlüssen ansetzen. Es ist nun aber eine Erfahrungstatsache, dass 2 Maxima gewöhnlich steil einfallenden Kluftscharen entsprechen, die dritte flach einfallenden Scharen. Die letzteren entsprechen gewöhnlich lithologischen Gegebenheiten, die ersteren werden als durch das geotektonische Spannungsfeld erzeugt angenommen. Daher sind es die rezenten steil einfallenden Klüfte, welche mit Bezug auf die Neotektonik von Interesse sind. Insofern, als man meistens zwei steil einfallende Kluftscharen erkennen kann, kann man diese als konjugierte Bruchflächen eines dreiachsigen Spannungszustandes betrachten (Mohr-Anderson-Theorie; ANDERSON, (1952). Aus der Stellung der Bruchflächen kann man daher die Hauptachsenrichtungen des neotektonischen Spannungszustandes rekonstruieren. Die gesamte Prozedur des Verpassens von 2 oder 3 Dimroth-Watson-Verteilungen und des Berechnens der Hauptspannungsrichtungen wurde für eine elektronische Rechenmaschine programmiert; notwendig ist lediglich die Eingabe der im Felde gemessenen Kluftorientierungen in numerischer Form! Die Darstellung der Messungen geschieht in der Weise, dass Flächen (Klüfte) jeweils durch ihre Fallrichtung (Azimut $\mathrm{N} \rightarrow \mathrm{E}$ in Grad) und Fallwinkel

Prof. Dr. Adrian E. Scheidegger, Technische Universität Wien, Institut für Geophysik, Gußhausstraße 27-29, A-1040 Wien (Austria) 
(Grad zur Horizontalen) fixiert werden. In graphischen Darstellungen werden Flächen durch ihren Pol auf einer flächentreuen (LAMBERT) Projektion der unteren Hälfte (plus $10^{\circ}$ ) einer Einheitskugel dargestellt. Gerade Linien (Richtungen) werden durch das Azimut ihrer Projektion auf eine Horizontalebene $(\mathrm{N} \rightarrow \mathrm{E}$ in $\mathrm{Grad})$ und ihre Neigung zur Horizontalebene (Grad) dargestellt; dabei entspricht das Azimut dem Absteigen der Geraden in den Untergrund. Punkt-dichten (gewöhnliche Poldichten) auf der Einheitskugel werden nach MÜLLER (1958) -Prozent gewertet.

Die Darstellung der Arbeiten ist standardisiert. Für jedes «Gebiet» geben wir die Lage der Messstellen, dann folgt eine ganz kurze Beschreibung der Geologie. Zuweilen fügen wir Fotografien typischer Aufschlüsse bei.

Die Rechenresultate werden dann für jedes Gebiet als Gesamtes gegeben. Dabei wird zu unterscheiden sein, ob die Messdaten Schicht- oder Sedimentations (S-)-Flächen enthalten oder nicht. Wenn sie sie enthalten, werden 3 Maxima aufgeführt, sonst nur 2. Falls sich in einem Gebiet die S-Flächen sehr stark verändern, müssen sie für die Ermittlung des $\mathrm{Ge}$ samtbildes einer Region eliminiert werden. In den Tabellen werden die numerischen Resultate in der Weise aufgeführt, dass für die 3 bevorzugten Kluftscharen (Max. 1, Max. 2 und Max. $3=\mathrm{S}$ ) jeweils die Fallrichtung $(\mathrm{N} \rightarrow \mathrm{E})$ und der Fallwinkel gegeben wird. Dann wird der Winkel zwischen Max. 1 und Max. 2 gebildet und schliesslich werden die Hauptspannungsrichtungen $\quad(\mathrm{P}=$ grösste,$\quad \mathrm{T}=$ kleinste Druckspannungsrichtung) angegeben. Zur Veranschaulichung des Gesamtbildes ist auch jeweils ein Poldichtediagramm (MÜLLER-Prozent) für die ganze Region beigefügt.

\section{Die untersuchten Gebiete}

\section{Allgemeines}

Die untersuchten Gebiete befinden sich in den Kantonen Bern, Aargau, Uri, Schwyz, Zürich, St. Gallen und Graubünden. In jedem untersuchten Gebiet wurden um einen "Schwerpunkt» eine Reihe von Aufschlüssen angesehen; die Lage dieser «Schwerpunkte» ist in Abb. 1 veranschaulicht. Geologisch reichen diese Gebiete von den Hochalpen bis zum Mittelland. Es waren auch zwei Bergsturzgebiete dabei (Amden und Goldau). Sie sollen nun vorerst einzeln, dann zusammen behandelt werden.

\section{Panixer Pass}

Beim Abstieg von der Panixerpasshöhe nach Rueun wurden an fünf Stellen Klüfte gemessen. Die Messstellen befanden sich in der Nähe des Saumpfades, zuerst an der Passhöhe $(2400 \mathrm{~m})$, dann bei $2200 \mathrm{~m}$, $1540 \mathrm{~m}$ und $1230 \mathrm{~m}$ Höhe.

Geologisch war man auf der Passhöhe in Kalk (Abb. 2), beim Abstieg folgte dann Schiefer (Verrucano, Perm) mit Kalklagen und schliesslich wieder Kalk. An jedem der erwähnten Aufschlüsse wurden je 21-22 Kluftmessungen durchgeführt. Schichtflächen wurden nicht aufgenommen. Somit ergaben die Rechnungen jeweils zwei signifikante Maxima, die untereinander recht konsistent waren. Das Gesamtbild der Region ist in der Tabelle 1 aufgeführt. Abb. 3 zeigt das Poldichtediagramm.

\section{Amden}

Eine Reihe von Kluftmessungen wurde in der Umgebung von Amden durchgeführt. Die Lage der Messstellen (mit 1-6 und A-H bezeichnet) ist aus Abb. 4 ersichtlich. Dieses Gebiet ist deshalb von besonderem Interesse, weil sich daselbst am 21. Januar 1974 ein Bergsturz ereignete, der sich fast zwei Jahre lang vorbereitet hatte. Aus diesem Grund soll dieses Gebiet auch etwas ausführlicher als die anderen behandelt werden. Das eigentliche Bergsturzgebiet befand sich westlich von Amden; der Abbruch erfolgte ungefähr beim in der Abb. 4 mit «C» bezeichneten Punkt.

Geologisch besteht das Bergsturzgebiet aus Schrattenkalk (Abb. 5, Stelle C), darunter liegen Drusbergschichten und Kieselkalk (Stellen G, H). Um jedoch die Untersuchung etwas umfassender zu gestalten, wurden Klüfte auch weiter im Norden gemessen: Am Mattstock (Stellen A und B sowie 4-6; Kalk der unteren Kreide) und am Speer (Stellen 1-3; Kalknagelfluh aus der unteren Süsswassermolasse, Abb. 6).

An den 14 Aufschlüssen wurden jeweils (ausser an einem) etwas über 20 Orientierungen gemessen. Dies ist zu wenig, um drei Dimroth-Watson-Verteilungen auf die Messwerte zu legen. Es wurden daher die Einzelaufschlüsse (ausser Aufschluss D, wo 33 Orientierungen gemessen worden waren) mit je zwei Verteilungen gerechnet. Die Rechenresultate sind in Tab. 2 gezeigt. 
Abb. 1: Lageplan der Messgebiete in der Schweiz Die Zahlen beziehen sich auf die Numerierung der Gebiete in Tab. 1

Abb. 2: Kalk auf der Panixerpasshöhe

Abb. 3: Poldichtediagramm für den Panixerpass

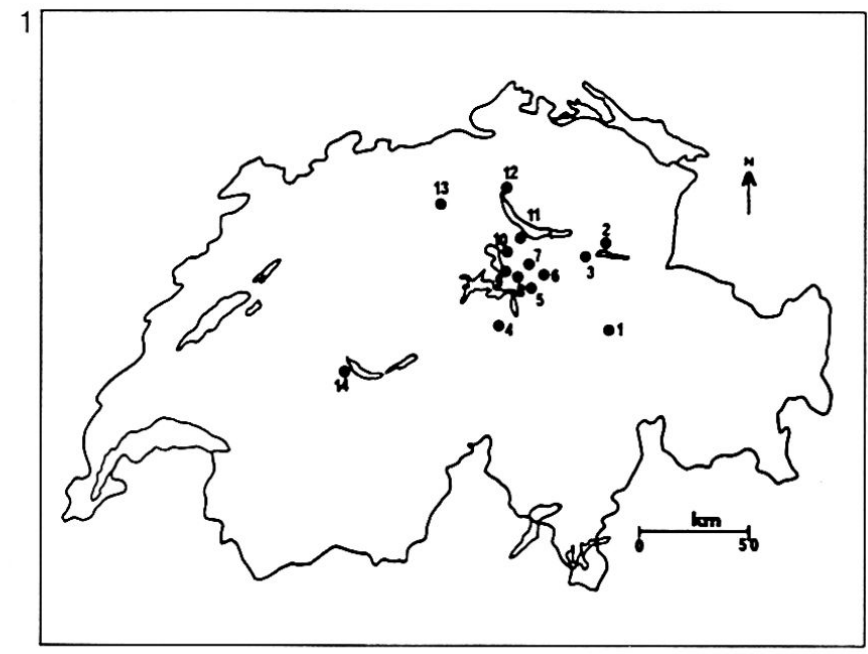

2
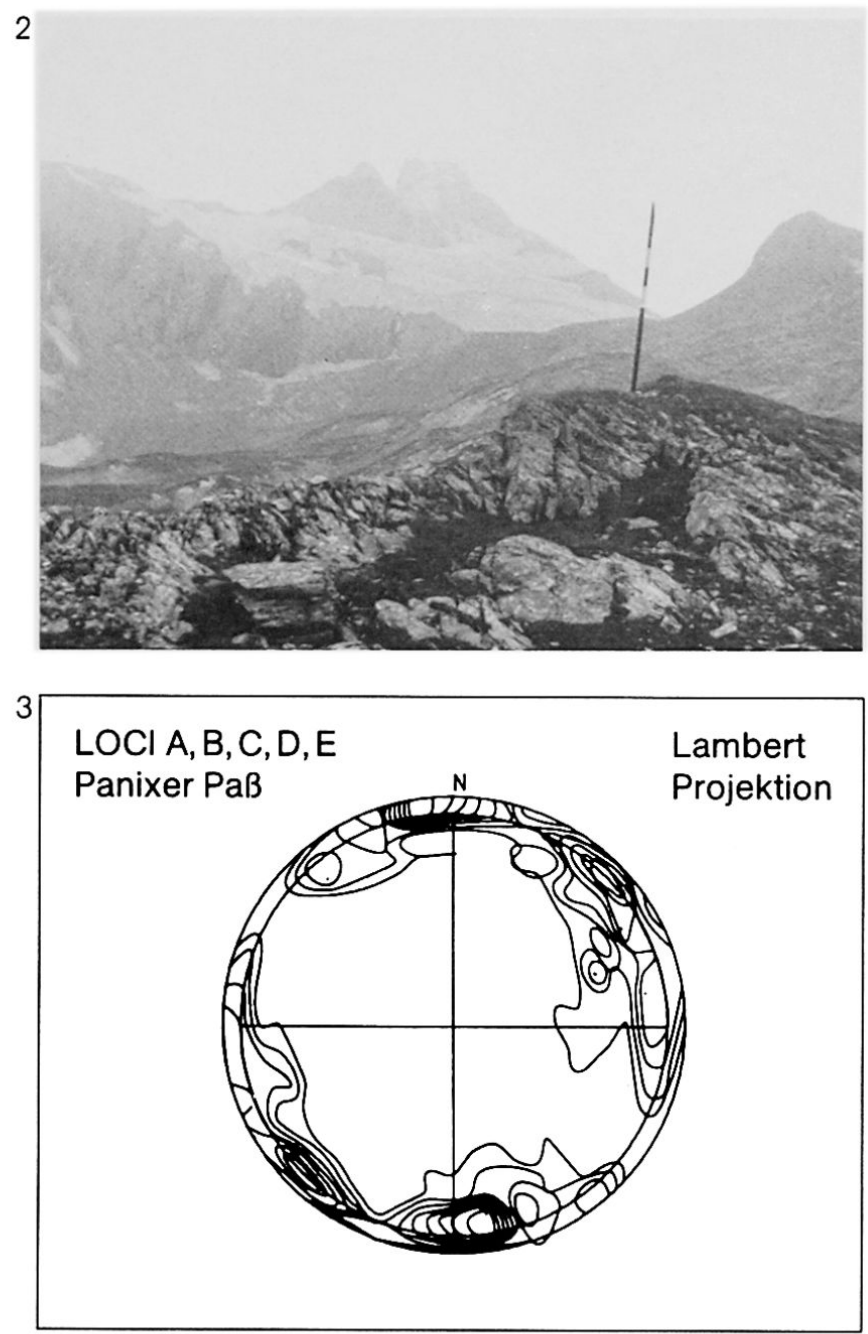

Abb. 4: Lageplan der Messstellen bei Amden

Abb. 5: Schrattenkalk des Bergsturzgebietes bei Amden
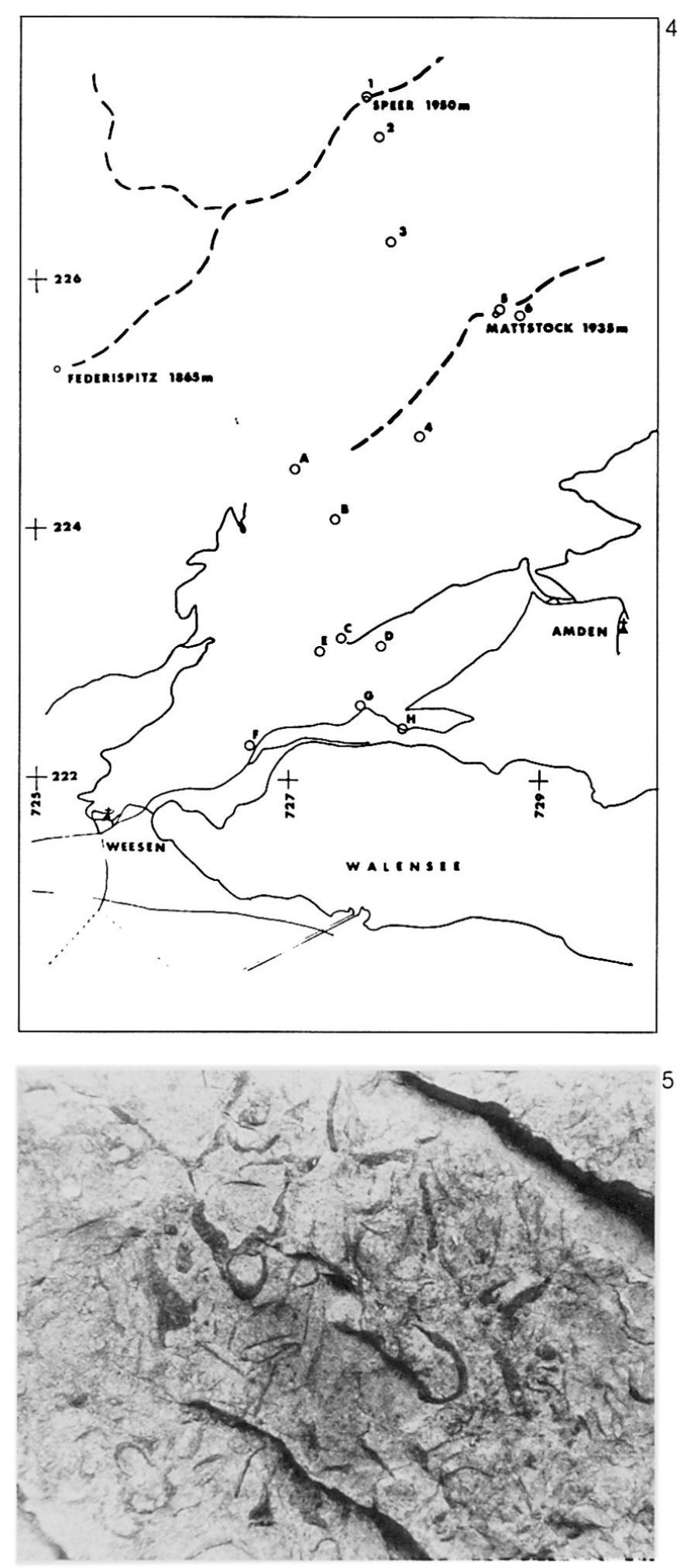
Abb. 6: Die (Speer)-Kette

Abb. 7: Hauptspannungsrichtungen in den Aufschlüssen bei Amden

6
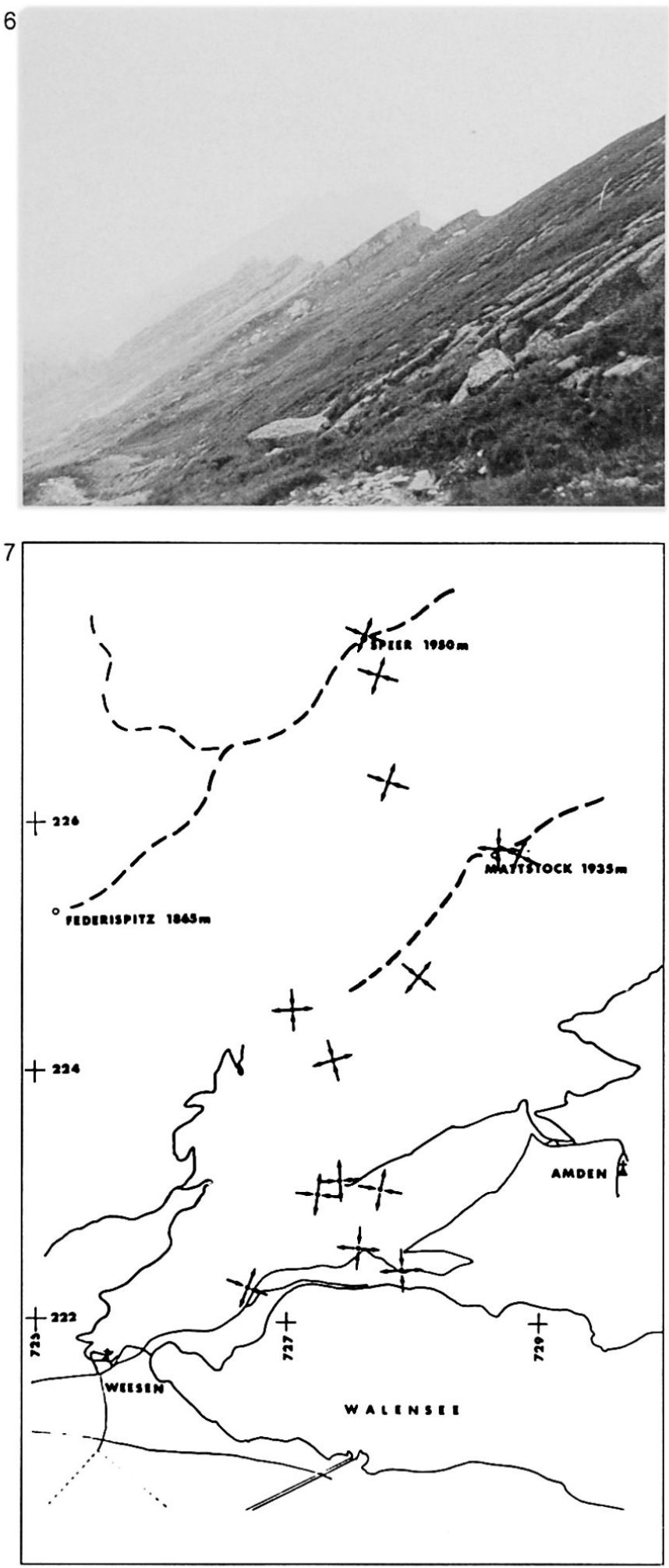

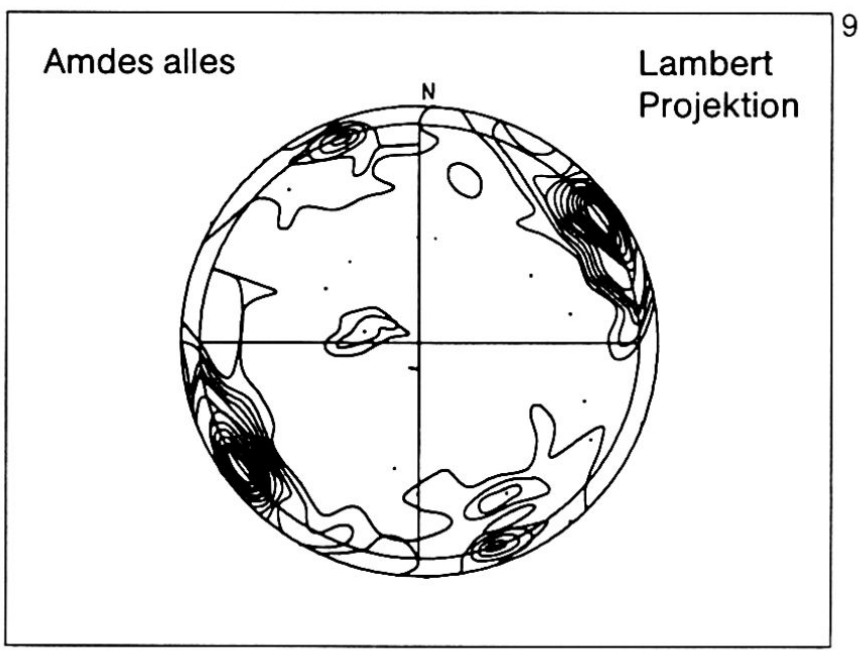


Die Messwerte enthalten aber einige Schichtungen, welche keineswegs horizontal sind (vgl. Abb. 6!); diese wurden speziell zusammengenommen, um eine mittlere Schichtorientierung der ganzen $\mathrm{Ge}$ gend zu erhalten (Wert für "S» in der Tabelle). Die Messungen sind detailliert genug, dass sich für das besprochene Gebiet die Einzelresultate aufarbeiten lassen. Abb. 7 zeigt eine Auftragung der gefundenen Hauptspannungsrichtungen an den individuellen Aufschlüssen. Durch diese lässt sich dann das in Abb. 8 gezeigte Netz von Spannungstrajektorien legen, wenn man die Identifizierung von $\mathrm{P}$ und $\mathrm{T}$ ausser Acht lässt. Hierzu ist aber zu bemerken, dass die WNW-ESE verlaufenden Trajektorien fast immer dem grössten Druck entsprechen, ausser an den Stellen 5, G und H. Die Stelle 5 (Mattstock Gipfel) zeigt sehr hohe Streuung und ist daher fragwürdig. Hingegen ist die Spannungsumkehrung in den Punkten $\mathrm{G}$ und $\mathrm{H}$ vermutlich signifikant: Der ganze $\mathrm{Ab}$ hang ist Rutschgebiet, welches vom Mattstock her gegen den See drückt. Wenn man daher die Wirkung von gravitationellen Kräften annimmt, müsste sich diese als eine maximale Druckspannung in der NS Richtung auswirken, was genau den aus den Klüften errechneten Spannungen entspricht. Die gefundene Umkehr des Vorzeichens der Spannungen entspricht also hier der Geomechanik des Amdener Bergsturzes.

Der Gesamtmittelwert des Gebietes "Amden" (alle Stellen) ist in Tab. 1 aufgeführt; Abb. 9 zeigt das Poldichtediagramm.

\section{Ziegelbrücke}

In recht enger Umgebung des Eisenbahnhofes Ziegelbrücke wurden an drei Stellen Klüfte gemessen. Die erste Stelle befand sich bei der westlichen Bahneinfahrt zum Bahnhof, die zweite über dem Tunnel bei der östlichen Einfahrt, und die dritte in der Mitte bei der Fussgängerbrücke.

Geologisch handelte es sich um Kalknagelfluh der unteren Süsswassermolasse (Abb. 10 [Oligozän bis Miozän]).

Bei den Kluftmessungen wurden keine Schichtungen aufgenommen. Somit handelt es sich prinzipiell um die Bestimmung von höchstens zwei Kluftscharen. Das Gesamtbild der Region ist in der Tabelle 1 angeführt. Abb. 11 zeigt das entsprechende Poldichtediagramm.

\section{Surenenpass}

Auf einer Wanderung von Attinghausen in Richtung Surenenpass wurden an vier Stellen Kluftmessungen durchgeführt. Alle Stellen befanden sich in der Nähe des Wanderweges von der Brüsti auf den Pass. Geologisch befand man sich an der tiefsten Stelle im Kalk (Abb. 12), darüber lag ein Sandstein der GaultFormation, weiter oben Schiefer und auf der Passhöhe Malm-Kalk mit Kalzitlagen.

Die Kluftdaten wurden jeweils mit 2 Dimroth-Watson-Verteilungen ausgewertet. Es wurden im Feld keine Schichtungen aufgenommen, und daher wurde bei drei Verteilungen das Maximum nicht mehr signifikant. Die Resultate für das Gesamtbild sind in Tab. 1 dargestellt. Es ergab sich eine bemerkenswerte Konsistenz zwischen den einzelnen Aufschlüssen. Abb. 13 zeigt das Poldichtediagramm der Gesamtregion.

\section{Mythen}

Die Mythen sind ein bekannter Gebirgsstock ob Schwyz (Abb. 14). Auf dem Abstieg vom Grossen Mythen Gipfel über die Holzegg und Zwüschet Mythen nach Schwyz wurden an vier Stellen Klüfte gemessen. Die Aufschlüsse befanden sich am Gipfel (1899 m) und am Weg auf den Höhen 1742 m, 1585 m und $1220 \mathrm{~m}$ über dem Meer.

Geologisch bilden die Mythen eine sogenannte «Klippe», d. h. eine Masse von Schichten, welche der näheren Umgebung vollkommen fremd sind. Die ältesten Schichten der Mythen besitzen ein Alter von etwa 200 Mill. Jahren, die jüngsten, nämlich die zu den Couches Rouges gehörenden, hellgrauen, schiefrigen Mergelkalke der Weissfluh, ein solches von etwa 70 Mill. Jahren. Die ganze Masse ruht jungen (etwa 50 Mill. Jahre alten), weichen, bräunlichgelben Mergelschiefern (Flysch) auf, welche die weiten Hänge gegen Schwyz hinunter bilden.

Bei den Kluftaufnahmen wurden pro Aufschluss etwas über 20 Orientierungen gemessen. Wir haben das Gesamtbild für alle Aufschlüsse berechnet. Man sieht nur zwei Richtungen deutlich. Abb. 15 zeigt das entsprechende Poldichtediagramm.

\section{Sihltalschluss}

Klüfte wurden an zwei Stellen im hinteren Sihltal südlich von Einsiedeln gemessen.

Die erste Stelle befand sich bei Untersihl. Es sind 
Abb. 10: Aufschluss bei Ziegelbrücke (Kalknagelfluh der unteren Süsswassermolasse [Oligozän bis Miozän])

Abb. 11: Poldichtediagramm für Ziegelbrücke

Abb. 13: Poldichtediagramm für den Surenenpass
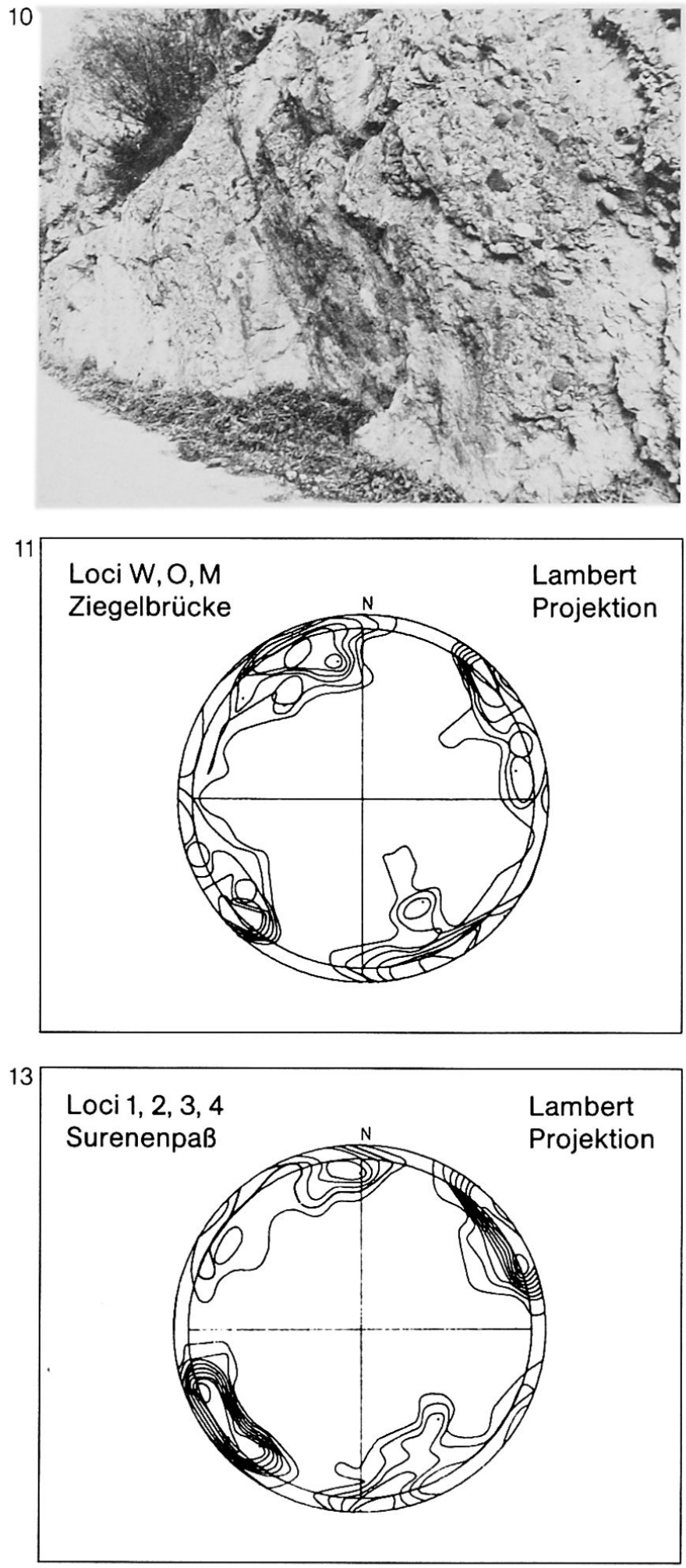
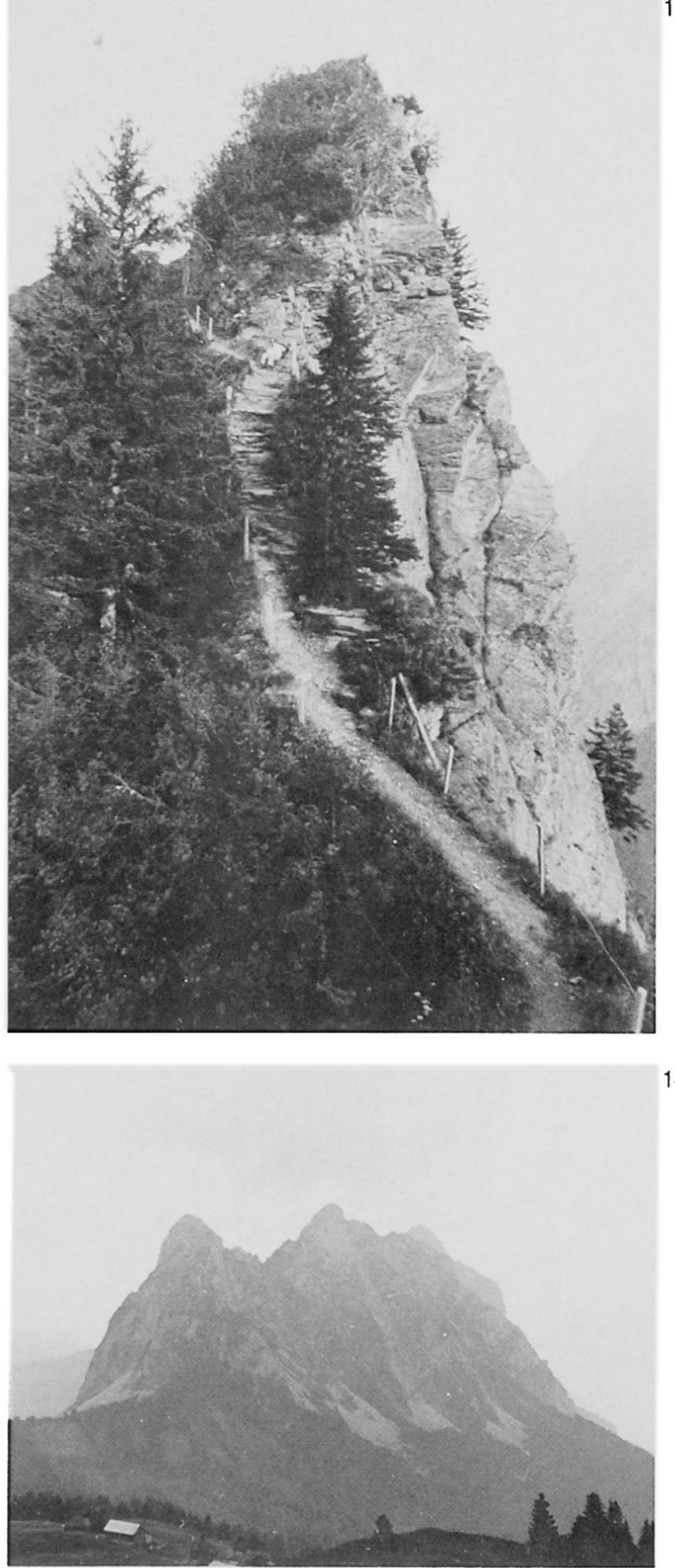
dabei schon im Hang bedeutende Klüfte sichtbar (Abb. 16). Das Material im Aufschluss selbst bestand aus Kieselkalk der unteren Kreide. Der zweite Aufschluss war weiter oben; hier waren ähnliche Kalke aus der Kreide aufgeschlossen.

Schichten wurden bei den Messungen nicht aufgenommen. Es waren daher in den Rechnungen nur zwei Maxima signifikant (Tab. 1). Man ersieht aus den Resultaten eine bemerkenswerte Konsistenz. Abb. 17 zeigt das Gesamtpoldichtediagramm für die Region.

\section{Umgebung von Einsiedeln}

Bei einer Wanderung, die von Einsiedeln über den Amselspitz und die Butzifluh nach Oberiberg führte, wurden unterwegs Klüfte an vier Aufschlüssen aufgenommen. Im Speziellen befindet sich die erste Stelle unterhalb der Alpe "Ufem Tritt», die zweite am Gipfel der Amselspitze, die dritte oberhalb der Butzifluh und die letzte im Surbrunnentobel.

Geologisch befindet man sich in diesem Gebiet in der Einsiedler Schuppenzone. Der erste Aufschluss zeigte Kalk, der zweite Aufschluss Schiefer und Kalkbänder, die Butzifluh besteht aus Nummulitenkalk und das Surbrunnentobel aus Wildflysch. Abb. 18 zeigt eine Aufnahme der Butzifluh.

Die Auswertung ergibt zwischen den ersten drei Aufschlüssen eine sehr gute Übereinstimmung. Der Aufschluss im Surbrunnentobel fällt dagegen etwas aus der Reihe. Nichtsdestoweniger wurden die Daten aller vier Aufschlüsse zusammengefasst, um ein regionales Bild zu erhalten. Dabei gehen die im Aufschluss im Surbrunnentobel vorhandenen Abweichungen in der Norm unter. Die numerischen Resultate sind in der Tabelle aufgeführt; Abb. 19 zeigt das Poldichtediagramm.

\section{Sattel - Schwyz}

In der Gegend von Sattel - Schwyz wurden Schichtund Kluftmessungen durchgeführt. Zwei Aufschlüsse befinden sich an der Strasse Sattel-Schwyz, zwei andere auf der Westseite des Tales oberhalb Sattel unterhalb des Wildspitzes in Kalk und Nagelfluh. Abb. 20 gibt ein Bild des Aufschlusses zwischen Sattel und Wildspitz (polygene Nagelfluh, Ober-Oligozän).

Die Messwerte wurden für die vier Messstellen numerisch nach unserer Methode statistisch ausge- wertet. Ein regionales Bild wurde dadurch ermittelt, dass alle Messstellen zusammengefasst wurden. Dabei braucht man die Schichtflächen nicht auszuschliessen, da sie relativ wenig streuen und daher eine regionale Schichtorientierung von $163 \pm 17 /$ $32 \pm 8$ ergeben. Das numerische Resultat für die zwei anderen Maxima ist in Tab. 1 aufgeführt. Abb. 21 zeigt das entsprechende Poldichtediagramm.

\section{Rossberg}

Am Rossberg wurden entlang der Rutschbahn des Goldauer Bergsturzes an drei Aufschlüssen Klüfte eingemessen.

Geologisch wechseln oligozäne Tone, Mergel und gelegentlich Sandsteinschichten mit mächtigen, festen, sehr regelmässig geklüfteten Nagelfluhbänken ab (HEIM, 1932). Ein typischer Aufschluss ist in Abb. 22 gezeigt (untere Süsswassermolasse).

Beim Messen der Klüfte wurden die sedimentären Schichtflächen auch mit aufgenommen. Obwohl diese manchmal etwas verwunden sind, fallen sie doch in der ganzen Gegend recht regelmässig gegen Süden zu und können daher auch über mehrere Aufschlüsse gemittelt werden. Bei der Auswertung muss man daher drei Dimroth-Watson-Verteilungen überlagern; das nach Süden fallende Maximum von $177 \pm 10 / 30 \pm 5$ repräsentiert auch die Schichtflächenschar, die zwei übrigen Maxima repräsentieren die tektonischen Klüfte. Die Resultate sind sehr wohldefiniert. Sie sind für das regionale Bild in Tab. 1 dargestellt. Abb. 23 zeigt ein Poldichtediagramm der Region.

\section{Oberägeri}

Klüfte wurden an zwei Stellen beim Übergang vom Finstersee nach Oberägeri gemessen. Die erste Stelle befand sich beim Gibel, die zweite auf dem Mangeli. Geologisch befanden sich diese Stellen in der unteren Süsswassermolasse. Der erste Aufschluss zeigte mergeligen Sandstein, der zweite (Abb. 24) Sandstein mit Nagelfluhbändern. (Oberoligozän: Höhrohnenschuppe.)

Auf dem Mangeli wurde ein Versuch gemacht, die Schichtung zu messen und $\mathrm{zu}$ identifizieren, sie streute aber sehr stark.

Das Gesamtbild der Region ist sonst recht konsistent. Das Resultat ist in Tab. 1 aufgeführt; Abb. 25 zeigt das Poldichtediagramm. 
Abb. 15: Poldichtediagramm für die Mythen

Abb. 16: Klüftungen am Hang des Sihltalschlusses

Abb. 17: Poldichtediagramm für den Sihltalschluss
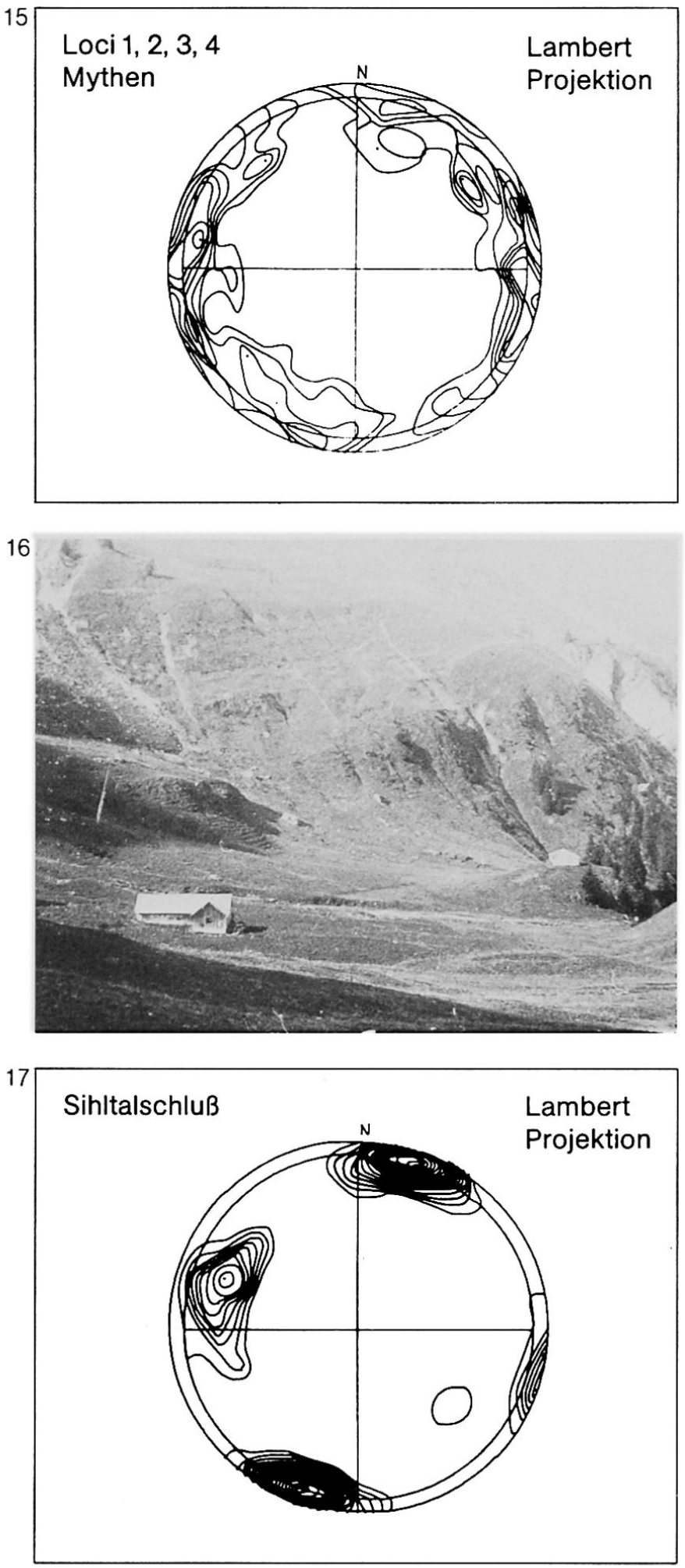

Abb. 18: Butzifluh

Abb. 19: Poldichtediagramm für die Umgebung von Einsiedeln

Abb. 20: Aufschluss an der Strasse von Sattel zur Kapelle am Wildspitz
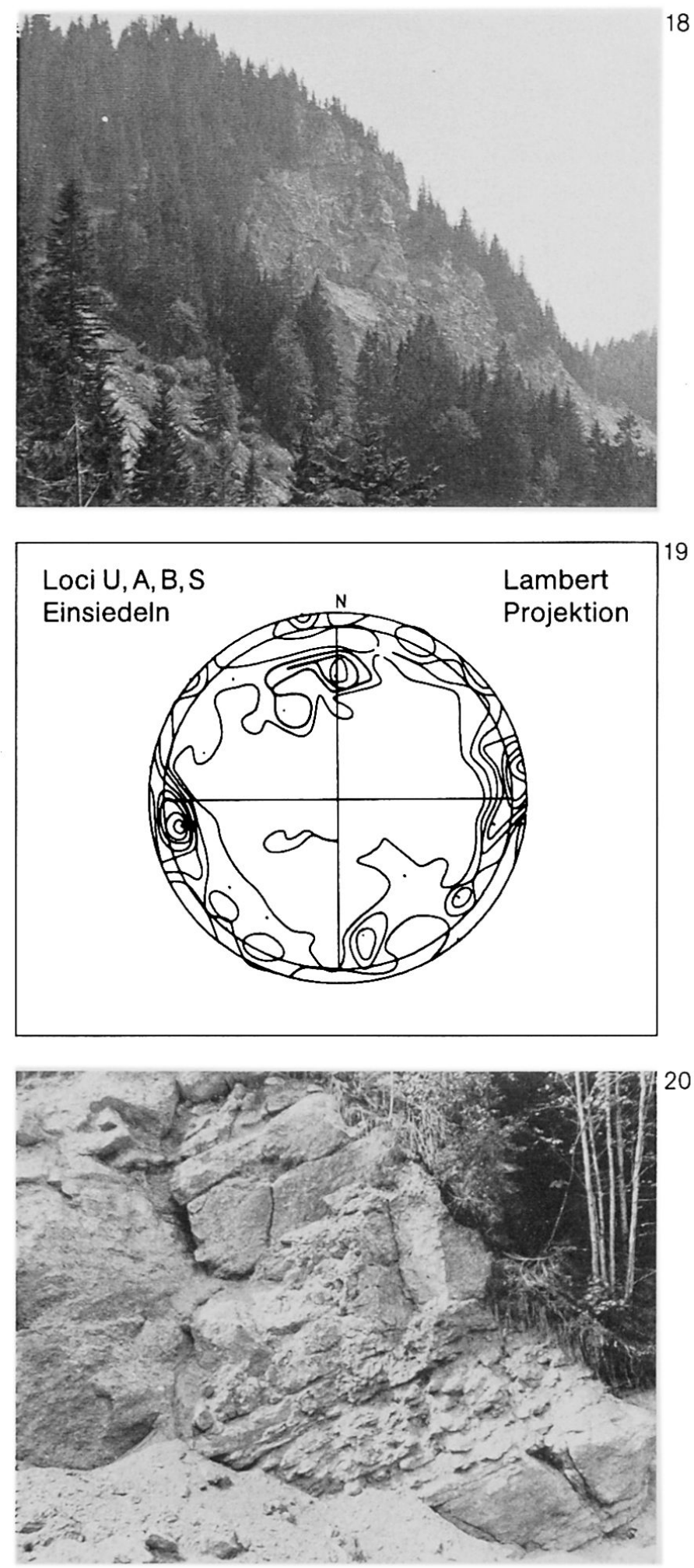
Abb. 21: Poldichtediagramm für die Region Sattel Schwyz

Abb. 22: Typischer Aufschluss am Rossberg

Abb. 23: Poldichtediagramm für den Rossberg
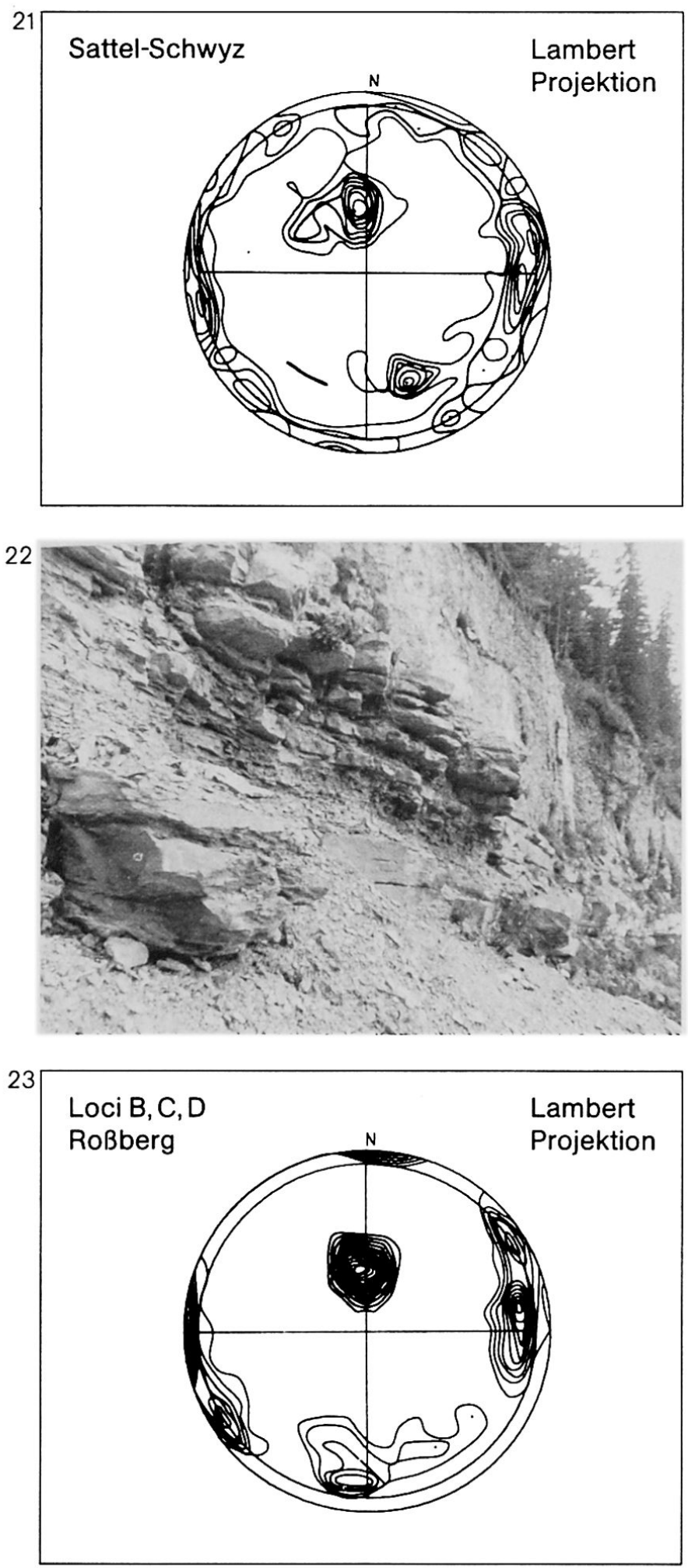

Abb. 24: Sandstein mit Nagelfluhbändern auf dem Mangeli (Oberoligozän: Höhrohnenschuppe)

Abb. 25: Poldichtediagramm Oberägeri

Abb. 26: Poldichtediagramm für Wädenswil
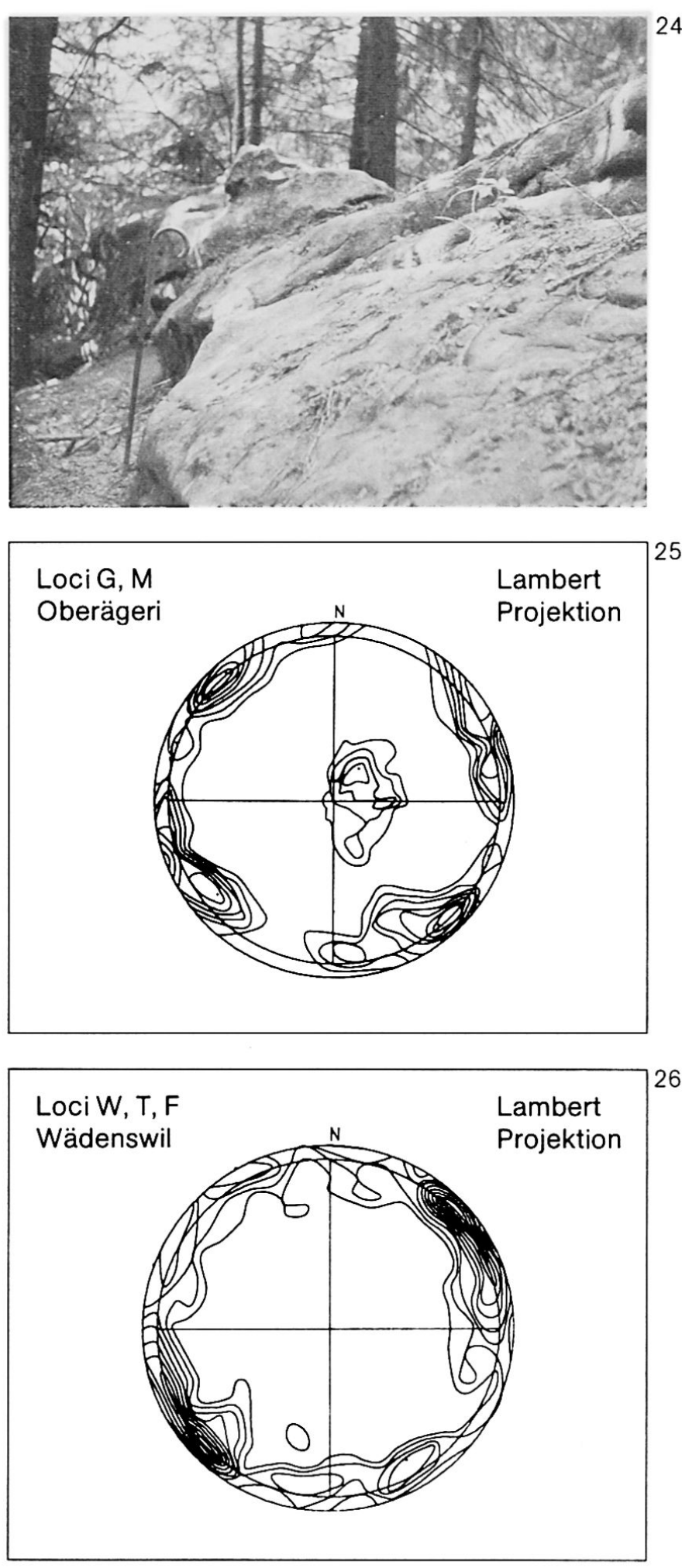


\section{Wädenswil}

Am Südufer des Zürichsees, in der Umgebung von Wädenswil, wurden Klüfte an drei Stellen gemessen. Die erste Stelle befindet sich beim Furthof, die zweite im Töbelibachtobel und die letzte bei der Ruine Wädenswil.

Geologisch handelt es sich hier um obere Süsswassermolasse. Die Aufschlüsse zeigen sehr mergelige Nagelfluh sowie Mergel mit Sandsteineinlagen, welche zu kleinen Wasserfällen Anlass geben. Die Ruine Wädenswil steht auf einem glazialen Rundhöcker (Kalknagelfluh).

Bei den Messungen wurden nur steilstehende Klüfte, keine Schichtungen aufgenommen. Daher wurden nur zwei Maxima für die Orientierungen vorausgesetzt. Die Resultate sind in Tab. 1 gezeigt. Abb. 26 gibt das Poldichtediagramm für diese Gegend.

\section{Zürich}

In der Umgebung von Zürich wurden auf dem Zürichberg Kluftmessungen durchgeführt. Die Messstellen befanden sich im Sagentobel und auf dem Loorenkopf.

In beiden Aufschlüssen fand sich obere Süsswassermolasse. Im Sagentobel waren es Tonschiefer, die mit Sandsteinlagen abwechselten: die letzteren geben jeweils Anlass zu Wasserfällen. Auf dem Loorenkopf (Abb. 27) befindet sich eine Nagelfluhkuppe, die einen glazialen Rundhöcker darstellt. Bei den Messungen wurden keine Schichtungen (SFlächen) aufgenommen. Daher wurden in der Darstellung der Messresultate jeweils nur zwei Maxima berechnet (Tab. 1).

Aus den Resultaten ersieht man, dass das Bild der Gesamtregion sehr bestimmt ist. Das Poldichtediagramm ist in Abb. 28 gezeigt.

In der Gegend von Lenzburg wurden Kluftorientierungen an zwei Aufschlüssen eingemessen. Der eine befindet sich am Schlosshügel Lenzburg, der andere am Rain bei Hendschiken. In beiden Fällen handelt es sich um der Molasse zugehörige Nagelfluh. Abb. 29 zeigt einen Anblick des Aufschlusses auf dem Schlosshügel Lenzburg.

\section{Gegend von Lenzburg}

Die numerisch berechneten Kluftorientierungsmaxima für die Messwerte sowie die dazu gehörigen
Hauptspannungsrichtungen sind in Tab. 1 aufgeführt. Das Gesamtbild für die Region wird auch als Poldichtediagramm gezeigt. (Abb. 30).

\section{Altes Kandertal}

Am Alpenrand, im alten Kandertal südlich von Thun, wurden Schicht- und Kluftmessungen durchgeführt. Ausser einem Aufschluss handelte es sich um Liaskalke (Klippendecke); ein einziger Aufschluss zeigte glazialen Deckenschotter, der zum Teil zu Nagelfluh verhärtet war.

Das regionale Kluftnetz wurde unter Weglassung der Schichtung berechnet. Das numerische Resultat ist in Tab. 1 dargestellt. Das entsprechende Poldichtediagramm ist in Abb. 31 gezeigt; dieses enthält die S-Werte.

\section{Diskussion}

\section{Numerische Zusammenfassung}

Die gefundenen Resultate wurden in Tab. 1 gesammelt und zusammenfassend dargestellt. Man sieht dabei, dass sich alles in allem eine bemerkenswerte Konsistenz für die untersuchten Gebiete der Schweiz ergibt.

Es ist daher vernünftig, einen Gesamtmittelwert für die Schweiz zu errechnen. Man kann dabei nicht alle Einzelkluftmessungen einfach gemeinsam behandeln, weil gewisse Lokalitäten (wie Amden) viel intensiver behandelt wurden als andere. Es wurden daher die errechneten Mittel-Werte für jede Region als «Input»-Daten für die Berechnung eines Gesamtmittelwertes verwendet. Das Resultat ist auch in Tab. 1 aufgeführt. Abb. 32 zeigt das entsprechende Poldichtediagramm.

\section{Kluftorientierungen}

Betrachten wir vorerst die Kluftorientierungen. Zur besseren Veranschaulichung wurden die Streichrichtungen der letzteren in den "Schwerpunkten»" auf eine Landkarte eingetragen (Abb. 33). Man sieht, dass die Mittelwerte, ausser bei den Mythen und im Sihltalschluss, einander mehr oder weniger parallel sind. Bei den Mythen, die als "Klippe» auf dem übrigen Gesteinsverband «sitzen», ist dies vielleicht nicht allzu erstaunlich, doch ist immerhin zu bemerken, dass die Richtungen bei den Mythen und im Sihltalschluss miteinander konsistent sind. Es sieht 
Tabelle I Gruppenwerte Schweiz

\begin{tabular}{lrrrllr}
\hline Locus & Zahl & \multicolumn{1}{l}{ Max. 1 } & Max. 2 & Winkel & P & T \\
\hline 1. Panixerpass & 106 & $244 \pm 9 / 83 \pm 8$ & $344 \pm 9 / 84 \pm 8$ & 80 & $114 / 10$ & $24 / 0$ \\
2. Amden & 320 & $57 \pm 4 / 90 \pm 4$ & $330 \pm 9 / 87 \pm 8$ & 87 & $103 / 2$ & $193 / 2$ \\
3. Ziegelbrücke & 65 & $60 \pm 11 / 89 \pm 10$ & $156 \pm 11 / 84 \pm 10$ & 84 & $288 / 5$ & $18 / 2$ \\
4. Surenenpass & 85 & $56 \pm 6 / 88 \pm 6$ & $336 \pm 11 / 88 \pm 10$ & 80 & $286 / 0$ & $196 / 3$ \\
5. Mythen & 98 & $26 \pm 19 / 83 \pm 13$ & $98 \pm 14 / 86 \pm 10$ & 72 & $152 / 2$ & $242 / 7$ \\
6. Sihltalschluss & 42 & $105 \pm 11 / 73 \pm 10$ & $196 \pm 5 / 89 \pm 5$ & 89 & $332 / 12$ & $240 / 11$ \\
7. Einsiedeln & 85 & $76 \pm 13 / 86 \pm 10$ & $342 \pm 26 / 90 \pm 19$ & 86 & $209 / 3$ & $299 / 3$ \\
8. Sattel-Schwyz & 128 & $257 \pm 15 / 85 \pm 11$ & $337 \pm 18 / 82 \pm 16$ & 80 & $207 / 3$ & $117 / 8$ \\
9. Rossberg & 95 & $259 \pm 11 / 84 \pm 10$ & $355 \pm 15 / 70 \pm 12$. & 86 & $126 / 19$ & $219 / 10$ \\
10. Oberägeri & 54 & $63 \pm 13 / 88 \pm 12$ & $326 \pm 14 / 84 \pm 13$ & 84 & $195 / 6$ & $104 / 2$ \\
11. Waedenswil & 70 & $64 \pm 13 / 89 \pm 10$ & $345 \pm 20 / 88 \pm 14$ & 79 & $115 / 0$ & $205 / 2$ \\
12. Zürich & 42 & $256 \pm 12 / 89 \pm 11$ & $170 \pm 14 / 87 \pm 12$ & 86 & $303 / 1$ & $33 / 3$ \\
13. Lenzburg & 45 & $249 \pm 11 / 89 \pm 11$ & $346 \pm 9 / 87 \pm 9$ & 83 & $118 / 3$ & $208 / 1$ \\
14. Altes Kandertal & 140 & $89 \pm 14 / 86 \pm 10$ & $180 \pm 28 / 84 \pm 15$ & 90 & $314 / 6$ & $44 / 1$ \\
\multicolumn{1}{l}{ Gesamt } & - & $73 \pm 12 / 89 \pm 11$ & $347 \pm 12 / 87 \pm 11$ & 86 & $120 / 2$ & $209 / 3$ \\
\hline
\end{tabular}

Tabelle 2 Amden, Einzelwerte

\begin{tabular}{|c|c|c|c|c|c|c|c|}
\hline Locus & No. & Max. 1 & Max. 2 & Max. $3=S$ & Angle & $\mathbf{P}$ & $\mathrm{T}$ \\
\hline 1 & 25 & $233 \pm 18 / 80 \pm 18$ & $342 \pm 15 / 70 \pm 14$ & - & 76 & $106 / 23$ & $200 / 7$ \\
\hline 2 & 25 & $57 \pm 8 / 88 \pm 8$ & $159 \pm 20 / 84 \pm 20$ & - & 78 & $288 / 7$ & $18 / 2$ \\
\hline 3 & 24 & $62 \pm 6 / 85 \pm 6$ & $157 \pm 12 / 85 \pm 12$ & - & 85 & $290 / 8$ & $20 / 0$ \\
\hline 4 & 21 & $76 \pm 40 / 78 \pm 26$ & $190 \pm 11 / 67 \pm 10$ & - & 73 & $310 / 30$ & $44 / 6$ \\
\hline 5 & 21 & $239 \pm 10 / 90 \pm 10$ & $305 \pm 28 / 77 \pm 24$ & - & 66 & $183 / 12$ & $92 / 8$ \\
\hline 6 & 21 & $70 \pm 13 / 90 \pm 13$ & $163 \pm 11 / 87 \pm 11$ & - & 87 & $297 / 2$ & $27 / 2$ \\
\hline $\mathbf{A}$ & 22 & $221 \pm 20 / 89 \pm 18$ & $131 \pm 15 / 79 \pm 15$ & - & 89 & $356 / 8$ & $266 / 7$ \\
\hline B & 21 & $219 \pm 31 / 74 \pm 23$ & $93 \pm 20 / 8 \pm 19$ & - & 59 & $341 / 26$ & $66 / 4$ \\
\hline C & 21 & $220 \pm 14 / 90 \pm 13$ & $315 \pm 20 / 89 \pm 20$ & - & 85 & $87 / 1$ & $177 / 0$ \\
\hline D & $33(11 S)$ & $240 \pm 22 / 88 \pm 19$ & $337 \pm 20 / 86 \pm 17$ & $99 \pm 27 / 18 \pm 8$ & 83 & $109 / 5$ & $199 / 1$ \\
\hline $\mathbf{E}$ & 22 & $227 \pm 10 / 88 \pm 10$ & $325 \pm 26 / 84 \pm 26$ & - & 82 & $97 / 6$ & $186 / 3$ \\
\hline F & 22 & $63 \pm 8 / 88 \pm 7$ & $344 \pm 28 / 76 \pm 25$ & - & 79 & $112 / 10$ & $204 / 10$ \\
\hline G & 21 & $56 \pm 11 / 84 \pm 12$ & $322 \pm 25 / 73 \pm 22$ & - & 88 & $190 / 16$ & $98 / 7$ \\
\hline $\mathbf{H}$ & 21 & $226 \pm 10 / 88 \pm 10$ & $307 \pm 20 / 82 \pm 19$ & - & 82 & $177 / 4$ & $86 / 7$ \\
\hline $\mathbf{S}$ & 16 & - & - & $106 \pm 37 / 18 \pm 11$ & - & - & - \\
\hline
\end{tabular}

also so aus, als ob hier eine lokale Anomalie bestünde.

Man kann versuchen, durch die Streichrichtungen der Klüfte ein Netz von Linien zu zeichnen; es ergibt sich dann das in Abb. 34 gezeigte Bild. Die systematische Abweichung im Sihltalschluss- und Mythengebiet äussert sich dabei als eine Anomalie.

\section{Spannungsfeld}

Wir haben schon erwähnt, dass sich nach der MohrAnderson'schen Theorie das die Klüfte vermutlich hervorgebracht habende Spannungsfeld berechnen lässt.

Wir haben die jeweiligen Hauptspannungsrichtungen für jeden "Schwerpunkt" von Messungen auf 
Abb. 27: Aufschluss am Loorenkopf

Abb. 28: Poldichtediagramm für Zürich

Abb. 30: Poldichtediagramm für den Aargau
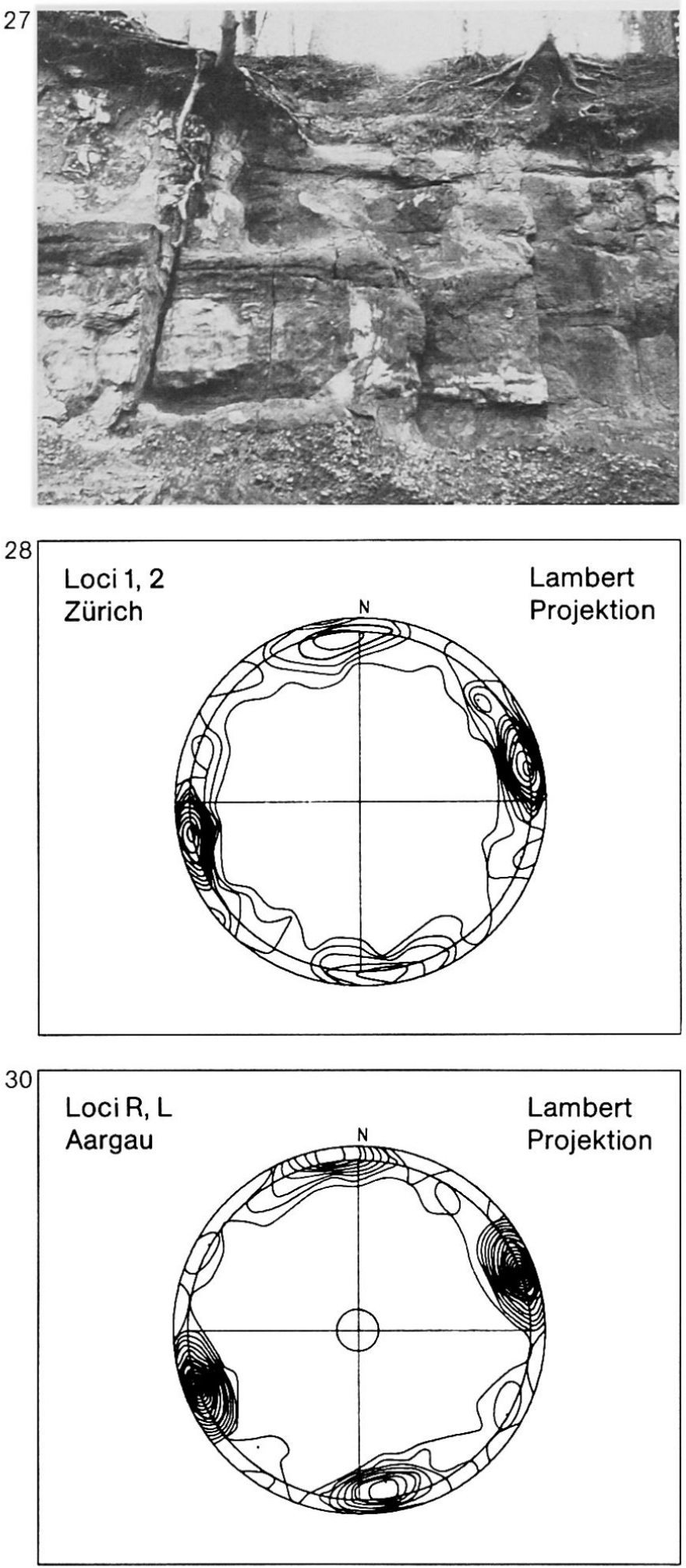

Abb. 29: Aufschluss am Schlosshügel Lenzburg

Abb. 31: Poldichtediagramm für das Alte Kandertal
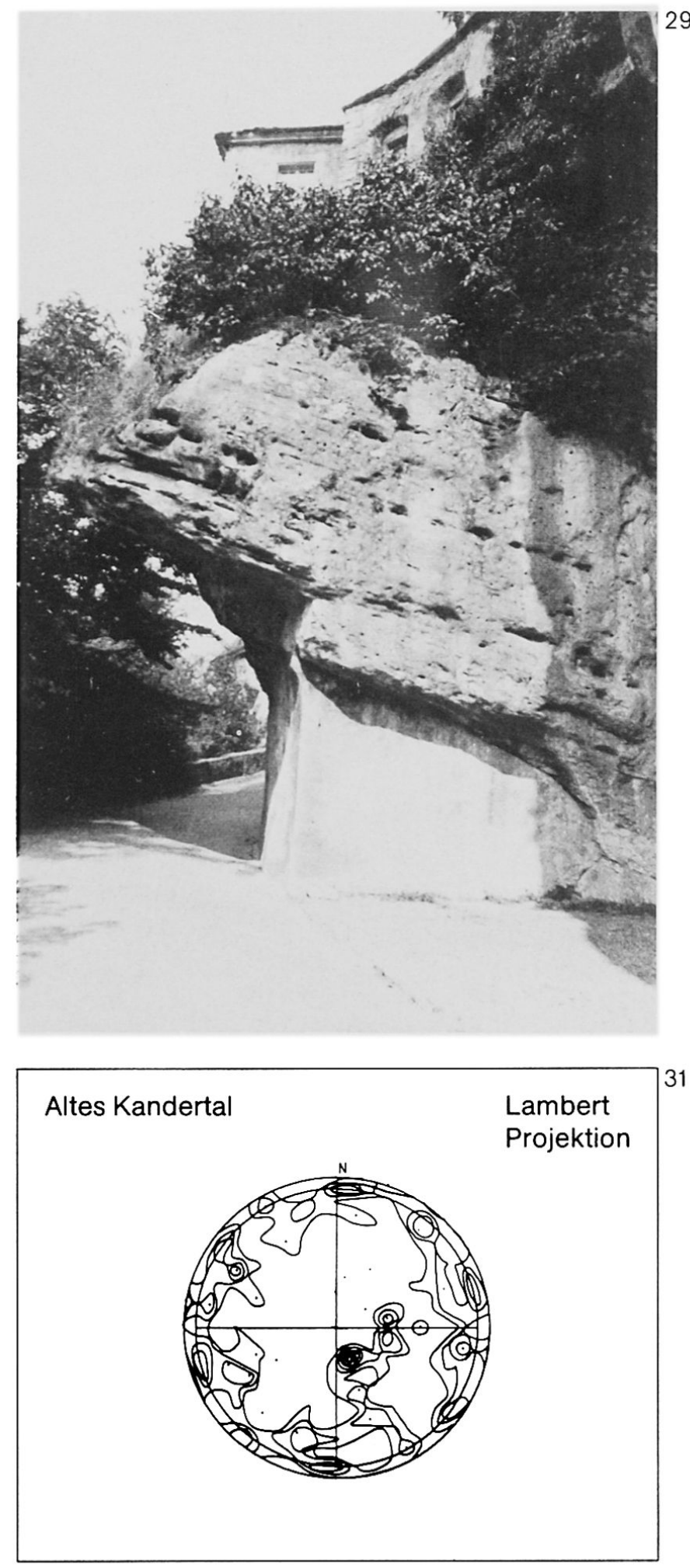
Abb. 32: Gesamtpoldichten für die Schweiz

Abb. 33: Gemittelte Kluftstreichrichtungen für die 14 Regionen der Schweiz

Abb. 34: Kluftnetz (Streichrichtungen) für die Schweiz
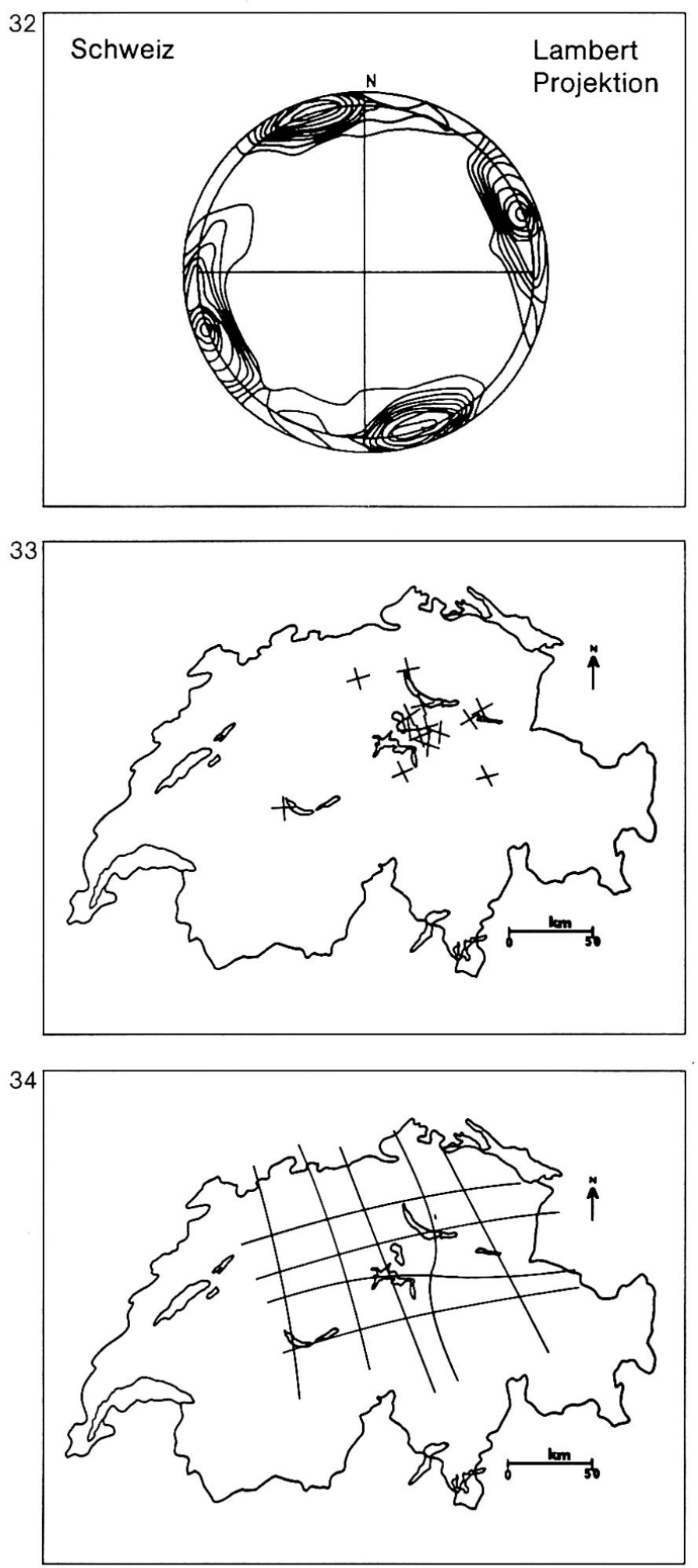

Abb. 35: Hauptspannungsrichtungen für die 14 Regionen der Schweiz

Abb. 36: Spannungstrajektorien für die Schweiz

Abb. 37: Vergleich der ermittelten grössten Druckspannungsrichtung von Gysel im Sonnenbergtunnel (insitu), von Greiner in Süddeutschland (in-situ) und durch den Autor (AES) aus Kluftmessungen
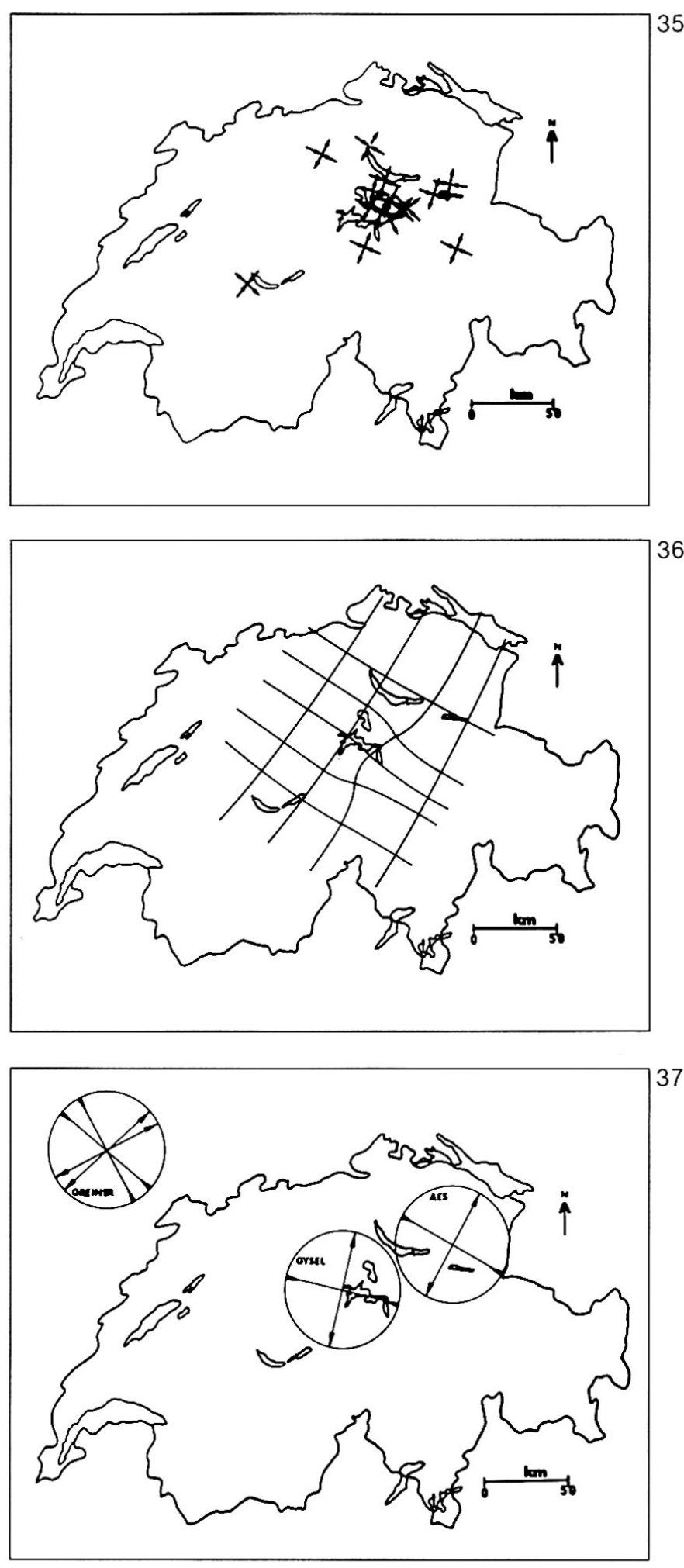
der Karte (Abb. 35) eingetragen. Wie bei den Klüften, erkennt man ein bemerkenswert konsistentes Bild, sogar wenn man die Identifizierung der grössten und kleinsten Druckspannung in Betracht zieht. Es gibt allerdings einige zusätzliche Diskrepanzen, wenn man das Spannungsvorzeichen in Betracht zieht; dabei muss man sich aber vergegenwärtigen, dass die Identifizierung von grösster und kleinster Druckspannung nie sehr sicher ist. Der Mittelwert für die grösste Druckspannung für das gesamte unsuchte Gebiet (Tab. 1) liegt bei N $120^{\circ} \mathrm{E}$. Man kann wiederum Trajektorien durch die Horizontalprojektionen der Hauptspannungsrichtungen legen und erhält dann das in Abb. 36gezeigte Bild. In der MythenSihltalschluss-Region entsteht dabei natürlich wiederum eine Anomalie.

Man kann die obigen Resultate mit in-situ Spannungsmessungen in der Gegend vergleichen. Abb. 37 zeigt das Resultat von GYSEL (1975), der bei Luzern (im Sonnenbergtunnel) Spannungen gemessen hat. In einem benachbarten Gebiet gibt es noch Resultate von GREINER (1975) für Messungen in Süddeutschland (Abb. 37). GYSEL gibt numerisch als grösste Druckspannungsrichtung im Sonnenbergtunnel bei Luzern die Normale zur Tunnelachse, $\mathrm{N} 103,5^{\circ} \mathrm{E}$ an. Die GREINERschen Werte von Süddeutschland liegen in der Schwäbischen Alb bei $\mathrm{N} 130^{\circ}-152^{\circ} \mathrm{E}$, in der Karlsruhe-Region bei $\mathrm{N} 140^{\circ} \mathrm{E}$ und in den Odenwald Bergen bei $\mathrm{N} 125^{\circ} \mathrm{E}$. Unser Mittelwert aus den Kluftmessungen gibt $\mathrm{N} 120^{\circ} \mathrm{E}$, also genau zwischen den Luzernern und den süddeutschen Werten. (Abb. 37).

Mit den obigen Resultaten kann man auch die Herdlösungen von Erdbeben in der Schweiz vergleichen (PAVONI, 1976), welche meistens eine Druckspannungsrichtung im NW-Quadranten ergeben und somit zu unseren Werten passen.

Man kann daher die Schlussfolgerung ziehen, dass die Kluftorientierungen in rezenten Aufschlüssen genau dem heutigen tektonischen Spannungsfeld, auch in geologisch komplizierten Gebieten, wie der Schweiz, durchaus entsprechen und dass umgekehrt die neotektonischen Hauptspannungsrichtungen aus Kluftorientierungen abgeleitet werden können.

\section{Dank}

Zur Beschreibung der Geologie der besuchten Gebiete haben Prof. Dr. R. Hantke in Zürich, Dr. E. Gerber in Schinznach und Dr. H. Buser in Schindellegi wesentlich beigetragen. Den beiden letzteren Herren verdankt der Autor auch die Führung auf mehreren Exkursionen. Bei den eigentlichen Messungen wurde der Schreibende in der Regel von seinem Sohn Paul assistiert.

Dem Rechenzentrum der Technischen Universität in Wien, Abteilungen Digitalrechenanlage und $\mathrm{Hy}$ bridenrechenanlage, gebührt Dank für die Bereitstellung von Rechenzeit und Dr.F. Kohlbeck in Wien für die Betreuung und Erstellung der Rechenprogramme sowie für das Stanzen vieler Datenkarten.

\section{Literatur}

ANDERSON, E. M. 1951: The Dynamics of Faulting and Dyke Formation with Applications to Britain. 2nd Ed., London: Oliver \& Boyd

GREINER, G.,1975: In-situ stress measurements in southwest Germany. Tectonophysics, 29: 265-274 GYSEL, M., 1975: In-situ stress measurements of the primary stress state in the Sonnenbergtunnel in Lucerne, Switzerland. Tectonophysics, 29: 301-314 HEIM, A., 1932: Bergsturz und Menschenleben. Zürich: Fretz \& Wasmuth A.G.

KOHLBECK, F., SCHEIDEGGER, A. E., 1977: On the theory of the evaluation of joint-orientation measurements. Rock Mechanics 9:9-25.

MÜLLER, L., 1958: Der Felsbau. Stuttgart: F. Enke Verlag

PAVONI, N., 1976: Herdmechanismen von Erdbeben und regional-tektonisches Spannungsfeld im Bereich der Geotraverse Basel-Chiasso. Schweiz. Min. Petr. Mitt. 56 (3): 